\title{
Epistemic transmission and interaction
}

\author{
L. Moretti • N. J. L. L. Pedersen
}

Received: 16 April 2013 / Accepted: 19 April 2013 / Published online: 14 May 2013

(C) Springer Science+Business Media Dordrecht 2013

Mainstream and formal epistemology naturally meet on issues pertaining to justification and knowledge transmission for one agent and cognitive interaction issues between two or more rational agents. Principles of transmission of justification, knowledge, and other epistemic properties have been subject to extensive investigation and discussion in the recent epistemological literature. Some discussions zoom in on epistemic transmission within a single agent. For instance, it has been hotly debated whether Moore's infamous proof of a material world transmits warrant from the premises to the conclusion. Some argue that it fails because one of its premises cannot be warranted prior and independently of the conclusion. More generally, it is an open issue whether the justification of perceptual belief transmits to all its logical consequences or whether it cannot transmit to the negations of the non-perceiving hypotheses (such as sceptical alternatives). Formal epistemologists have been investigating the conditions that permit incremental confirmation to transmit across entailment. Other discussions concern the transmission of true belief, warrant or knowledge between agents - the discussion of testimonial warrant and knowledge being a prime example, another being the debate over whether certain arrangements (such as free speech) do better in terms of promotion of true beliefs in groups of agents than alternative arrangements.

L. Moretti $(\bowtie)$

Philosophy Department, University of Aberdeen, Old Brewery, High Street, Aberdeen AB24 3UB, Scotland, UK

e-mail: 1.moretti@abdn.ac.uk

N. J. L. L. Pedersen

Underwood International College, Yonsei International Campus,

Veritas Hall B 427, 162-1 Songdo-dong, Yeonsu-gu,

Incheon 406-840, South Korea

e-mail: nikolaj@yonsei.ac.kr 
We do not live in complete epistemic isolation. We interact in epistemically significant ways with other agents. For example, we acquire justification and knowledge for many of our beliefs via testimony. Recently, there has been a surge of interest in the epistemic significance of disagreement - the main question being whether, and under what conditions, disagreement with others rationally requires one to suspend one's initial belief or lower one's initial credence. On the other hand, in interacting with others we also sometimes find ourselves agreeing with them. Under what conditions should the discovery of such agreement increase one's credence? Additionally, much contemporary formal modelling focuses on active agency and rational interaction among agents whether this interaction is to change beliefs collectively, process information in groups, learn and divide of the burden of epistemic labour or avoid various unpleasant epistemic phenomena ranging from pluralistic ignorance and informational cascades to belief polarization.

The aim of this special issue is to investigate themes and questions pertaining to the transmission of justification, knowledge, and other epistemic statuses and cognitive interaction between agents through applications of formal and informal methods. The contribution by L. Moretti and T. Piazza 'When warrant transmits and when it doesn't: towards a general framework' aims to provide necessary and sufficient conditions for transmission of different types warrant across entailment and to elucidate their epistemic benefits. The paper also scrutinises C. Wright's analysis of transmission failure, criticises it and suggests strategies to cope with some of its problems.

Within the standard possible worlds framework, agents are logically omniscient. This is known as the problem of logical omniscience. For, while fully ideal agents may be logically omniscient, ordinary agents are clearly not so. In order to circumvent the problem of logical omniscience some people introduce impossible worlds—worlds where logical truths can be false, or where 'anything goes'. However, this move delivers agents that are radically non-ideal in that they fail to perform even very simple logical inferences. This raises the question whether the possible worlds framework can be used to model moderately ideal agents - agents that fall short of logical omniscience, but are still somewhat logically competent. In 'Impossible worlds and logical omniscience: an impossibility result' J. C. Bjerring argues that this cannot be done.

The contribution by T. Shogenji 'Coherence of the contents and the transmission of probabilistic support' investigates how the coherence of the contents of evidence (e.g. the contents of testimonial reports) affects the transmission of probabilistic support from the evidence to a target hypothesis. Shogenji argues that when the coherence of the contents of evidence is measured in a specific way, it does reduce the transmission of probabilistic support from the evidence to the hypothesis. Other aspects of the relations among the contents may offset, however, this negative impact of coherence. Shogenji's work questions claimed consequences of important proofs in formal coherentism, such as L. Bovens and S. Hartmann's and E. Olsson's impossibility theorems.

The contribution by R. De Langhe 'Peer disagreement under multiple epistemic systems' aims at an explanation or justification of longstanding peer disagreement. Peers are usually assumed to share the same evidence. However-as A. Goldman has suggested - they might not share the same evidence for the epistemic system (i.e. sets of norms, standards, or principles for forming beliefs). De Langhe elaborates diachronically this possible complication of the peer disagreement debate by using a 
simulation. The interesting result (which extends previous work by I. Douven) is that the very possibility of multiple epistemic systems downgrades the epistemic value of consensus and renders difference splitting a suboptimal strategy to reach the truth.

Like De Langhe's contribution, E. J. Olsson and A. Vallinder's 'Norms of assertion and communication in social networks' concerns epistemic interaction, but with the focus moved from disagreement to assertion in the context of group deliberation. Olsson and Vallinder investigate what norm of assertion maximizes the epistemic good of truth. They do so in Laputa, a computer simulation developed by S. Angere and Olsson himself. On the basis of the simulation data they suggest that a norm that sets the threshold of assertion at certainty is optimal in the limit, but that a lower threshold better promotes truth in non-limit—or less idealized-cases.

We rely epistemically on others. Sometimes our reliance consists in us receiving information, while other times it consists in exactly the opposite: not receiving any information. As for the latter, we sometimes carry out absence-based inferences. This happens when the fact that no information to the effect that $\mathrm{P}$ is received from any source is used as a basis for inferring $\sim p$. S. Goldberg has discussed this kind of inference in detail. He argues that it is epistemically good (or conditionally reliable) provided that the agent enjoys epistemic coverage, meaning that the sources on which she is relying are sufficiently connected to the relevant domain of enquiry. N. J. L. L. Pedersen and J. Kallestrup's contribution 'The epistemology of absence-based inference' further discusses epistemic coverage and its epistemic significance. Pedersen and Kallestrup rework the notion of epistemic coverage and connect absence-based inference to the controversial issue whether absence of evidence amounts to evidence of absence. This is not generally so. However, they argue that, given epistemic coverage (and some additional conditions), absence of evidence does amount to evidence of absence.

Acknowledgments Some of the papers published in this issue were presented at the Epistemic Transmission and Interaction Workshop at the University of Copenhagen in October 2010. The workshop was generously funded by Vincent Hendricks' Elite Research Prize (awarded by the Danish Ministry of Science, Technology and Innovation) and the Northern Institute of Philosophy, University of Aberdeen. 